\title{
B2B E-Commerce Infrastructure Using Agents and Standards - A Potential Impact Analysis and Architecture
}

\author{
N. Ivezic
}

National Institute of Standards and Technologie, Oak Ridge National Laboratory, USA

Em: Nenad.Ivezic@nist.gov

L. Fong, T. Rhodes

National Institute of Standards and Technologie, USA

Y. Peng

University of Maryland at Baltimore County, USA

Keywords business-to-business, electronic commerce, agents, standards, business case

\begin{abstract}
Multi-agent systems are typically being developed independent of industrial standards. In this paper, we present an agent architecture for business-tobusiness electronic commerce in the domain of circuit board manufacturing that builds on a number of standards. Our architecture builds on the GenCAM standard from the circuit board manufacturing sector and RosettaNet from the B2B community.
\end{abstract}

\section{INTRODUCTION}

The Internet Commerce for Manufacturing (ICM) project is working with the printed circuit (PC) board and PC assembly (PCB/PCA) industries to extend and apply emerging Internet-based technologies in support of manufacturing. In this paper, we focus on the ICM project component that develops and evaluates agent technologies to address two PCB/PCA industry issues: the PC board manufacturing information exchange and the supply chain information integration. Differently from other approaches in developing agent systems, our agent approach attempts to use existing PCA/PCB industry standardization efforts. First, we rely on the emerging 
GenCAM standardization effort in support of PC board manufacturing information exchange [1]. Second, we use RosettaNet standardization specifications in support of supply chain information integration in the electronics component industry [2].

In the rest of the paper, first, we provide an assessment of potential impact of agent technologies within the realm of the Internet-based Commerce for Manufacturing (ICM) project. Following the assessment, we identify two areas of agent technology development that we pursue and describe the agent architecture for this work. Finally, in the last section, we summarize the value-added contribution of the agent technologies within the ICM domain of interest.

\section{AN IMPACT ANALYSIS FOR ICM PROJECT}

To date, the ICM project has looked closely at the manufacturing Business-to-Business (B2B) area as the primary domain of investigation. The rationale for this focus was the potential impact of the new Internetbased solutions in the emerging electronic markets of manufacturing goods. The team identified several alternative manufacturing functions and B2B use cases where agent solutions could take place. We have taken the identified opportunities for the agent-based technologies from B2B area and performed an evaluation of these opportunities, as they apply to the ICM domain. The metrics used to perform the evaluation were the potential impact, complexity, and relevance to the ICM area of interest. Table 1 gives a summary of the outcome of the evaluation process:

First, Flexible Customer-to-Supplier Interfaces, points at the opportunity for agent approaches to 'wrap around' or completely circumvent the existing form-based interfaces on the Web that have pre-defined syntax, implicit semantics, unpublished interaction protocols and, instead, enable automated, on-demand B2B interface construction.

Second, Optimized Negotiation of Service Cost and Terms, points to the fact that an expert human is solely responsible to negotiate 'optimal' set of terms and costs of service. This decision making approach requires an expert human, who, often has only a limited view of the business situation and cannot react immediately to new business situations. Agent approaches carry promise of embedding significant decision making capabilities and supporting the human in identifying the optimal choices.

Third, Efficient Intra-Enterprise Technology Adoption and Adaptation, points at the issue that currently, few means exist to accelerate adoption and adaptation of new e-commerce technologies within an enterprise. An opportunity exists for agent approaches to provide for easier integration with 
legacy systems through usage of shared languages and ontologies and efficient updates of interaction protocols.

Fourth, Efficient Engineering Change Order (ECO) Processing, indicates that, currently, processing ECOs require each human participant in the engineering and manufacturing process to manually sign-off on the change and to make sure that the change is appropriately reflected in the part of the process for which the human is responsible. Agent technologies hold potential to make this process much less tedious and error-prone.

Fourth, Efficient Inter-Enterprise Interaction Technology Support, indicates that currently, very few means exist to accelerate adoption of new B2B communication technologies across enterprises so that the enterprises can quickly engage in new inter-enterprise interactions. Agent solutions promise to automate such inter-enterprise interaction setup and support.

Table 1 Opportunities for Agent-based Internet Commerce for Manufacturing.

\begin{tabular}{llll} 
& Impact & Complexity & Relevance \\
\hline Flexible Customer-to-Supplier Interfaces & Med-High & Med & Med-High \\
\hline Optimized Negotiation of Service Cost and Terms & Med & Med-High & Low \\
\hline $\begin{array}{l}\text { Efficient Intra-Enterprise Technology Adoption } \\
\text { and Adaptation }\end{array}$ & Med-High & Med & Med-High \\
\hline $\begin{array}{l}\text { Efficient Engineering Change Order (ECO) } \\
\text { Processing }\end{array}$ & Med-High & Med-High & Med-High \\
\hline $\begin{array}{l}\text { Efficient Inter-Enterprise Interaction Technology } \\
\text { Support }\end{array}$ & Med-High & Med & Med-High \\
\hline
\end{tabular}

\section{AGENTS-BASED SOLUTIONS IN ICM PROJECT}

Based on the impact analysis in Section 3, we selected two areas of development for the agent technologies. The first area was the Flexible Customer-to-Supplier Interfaces where we focused on development of GenCAM information retriever agent to enable automated B2B interface construction. The second area was the Efficient Inter-Enterprise Interaction Technology Support to develop a supply chain integration agent technology in support of emerging B2B standards such as RosettaNet. The rationale for this selection is demonstrated in Table 1 - it was determined that we could achieve a significant impact through the technology development in the area that is very relevant to the ICM domain while managing the development complexity. Moreover, the GenCAM and RosettaNet standards are becoming mature to the degree that basing the agent technology on these standards seemed to be warranted. In the following, we use an example 
business-to-business situation (i.e., an RFQ scenario) to describe architectures of the agent technology developments.

\subsection{An Example Problem: RFQ Scenario}

In this RFQ scenario, an original equipment manufacturer (OEM), responsible for developing a $\mathrm{PC}$ board, issues a request for quotes to its suppliers - electronics manufacturing service (EMS) providers - to perform a specific service to build the PC board (e.g., bare board production, part supply, part preparation). The EMS provider requests additional information that is not provided within the technical package. The OEM searches for the additional information in the technical database, identifies the requested data, and provides the data back to the EMS provider.

Some of the EMS providers may provide a counter-offer with a portion of the original specification altered (e.g., time of delivery, quantity) and traded off (e.g., cost versus time of delivery). The OEM, than, may enter negotiation with one or more suppliers to determine completion of the service. A series of counter-offers may be exchanged until the process is completed at the time OEM selects the winning bids.

The scenario serves to demonstrate some of the principal traits of the following agent based solutions and is not meant to capture all peculiarities of the RFQ process.

\subsection{GenCAM Agent-based System Architecture}

Figure 1 shows the proposed architecture for an intra-enterprise information retrieval agent system supporting OEM RFQ functionality. The system is composed of four agent types. Inter-Enterprise Broker Agent (BA) is responsible for collecting advertisements from all system agents regarding their individual capabilities. Human-Computer Interaction Agent (HCIA) facilitates interaction between the human user and the agent system. Web Assistant Agent (WA) supports interaction between the agent system and the EMS client that consists of a human user with a Web browser. Finally, GenCAM Specialist Agent is responsible for retrieving information from the GenCAM technical database.

The proposed architecture addresses several issues in intra-enterprise information retrieval and in support of RFQ functionality. First, the GenCAM technical data standard for PC board manufacturing is becoming increasingly complex. By providing a wrapper agent capable of searching, retrieving, and reasoning about PC board data, the standard interface is 
localized and isolated from the rest of the system. Changes in the GenCAM standard and technical databases may be handled in a local fashion without affecting the remaining system. Second, the interface between the EMS user and the OEM agent system becomes simpler and more general by isolating the 'content' of transactions in the GenCAM agent. Only the GenCAM agent on the OEM side and a plug-in, a client within the Web browser, or an application program on the EMS side needs to understand the GenCAM content. The remaining agents of the OEM agent system need not to be concerned with the content.

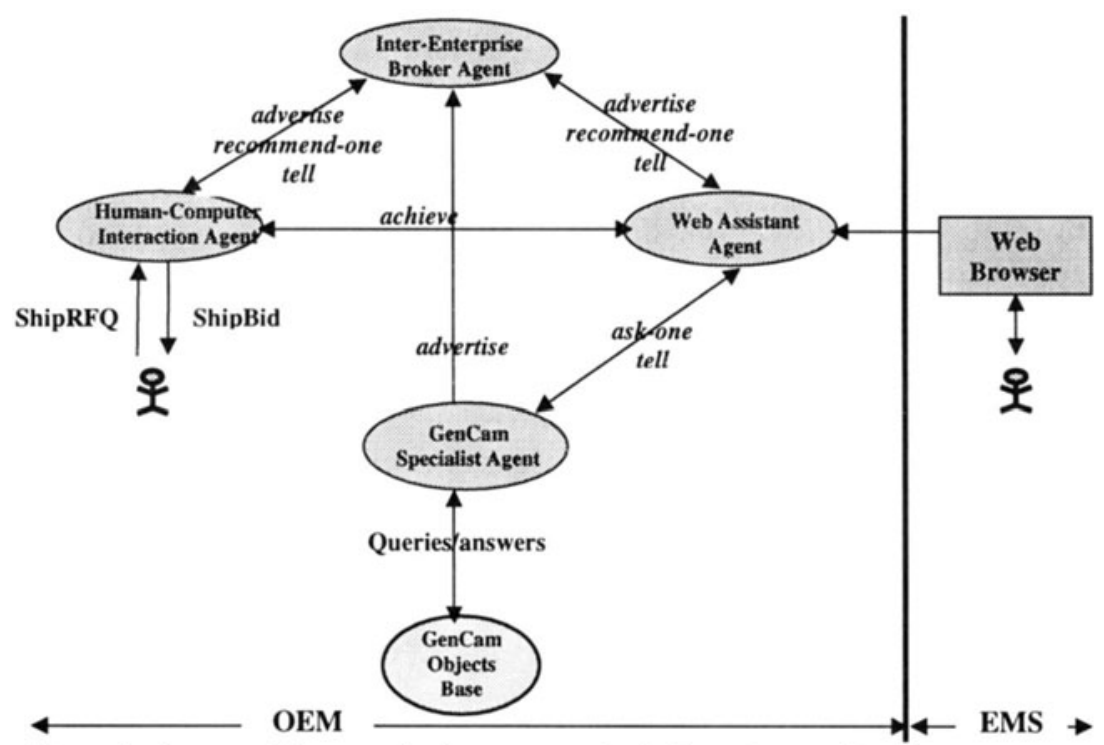

Figure 1 Agent architecture for intra-enterprise information retrieval agent system

While this agent architecture addresses the intra-enterprise information retrieval and interaction with a human on the side of the EMS client, a complementary supply chain integration system is described next that allows automated agent-based inter-enterprise interactions.

\subsection{RosettaNet Agent-based System Architecture}

Figure 2 illustrates the proposed architecture for an inter-enterprise supply chain integration system in support of RFQ scenario. The system is composed of two new agent types. Supply Chain Interaction Agent (SCIA) takes place of the Web Assistant Agent (WA) from the previous architecture to enable agent-based interaction among enterprises. The Inter-Enterprise Broker Agent (IEB) is responsible for matching advertised capabilities and 
requirements on part of agents in the supply chain. Within the EMS provider, there is an opportunity to introduce a Bidding Specialist Agent to automate the bidding process. A human user interacts with the system through the HCIA to check and approve the bids.

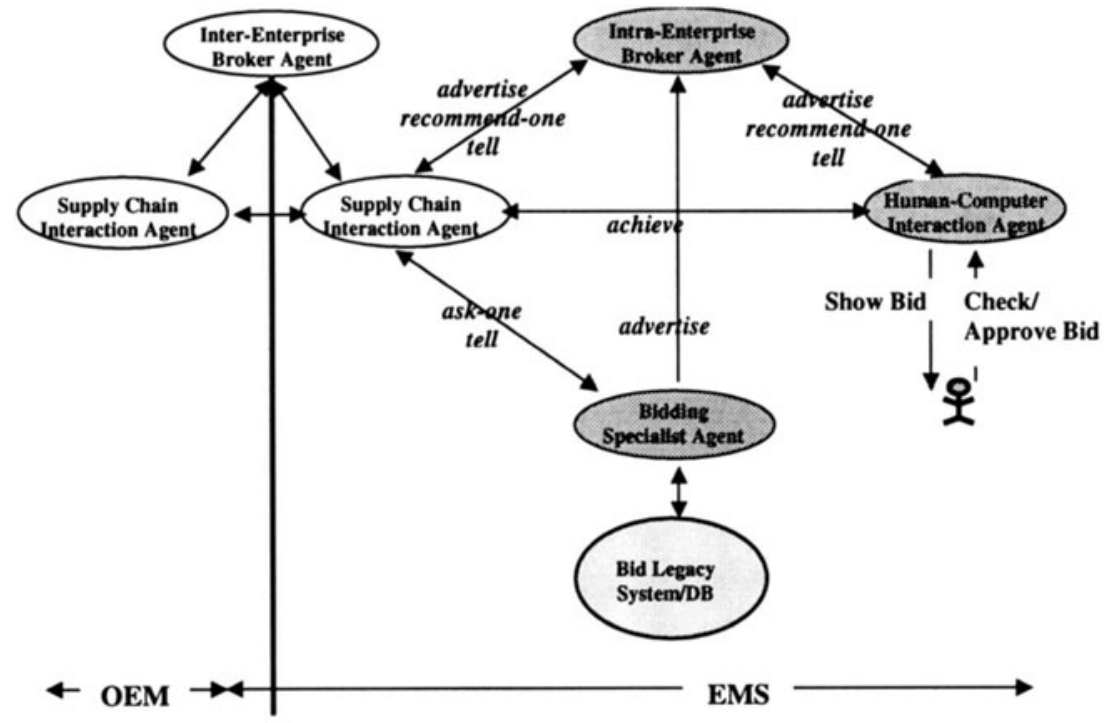

Figure 2 Agent architecture for inter-enterprise supply chain integration system

The supply chain integration architecture further provides for automation of interactions among OEMs and EMS providers. Our intent is to use the proposed architecture to map its coordination protocols onto emerging standards such as RosettaNet and provide approach to capture business-level interactions among customers and suppliers. Another goal is to merge the inter-enterprise and the intra-enterprise informational retrieval architecture to explore issues in supply chain integration standards development and harmonization.

\section{EXPECTED IMPACT OF ICM AGENTS SOLUTION}

There are four distinct value-added opportunities in adopting agentbased technologies within the ICM project:

First, an agent-based approach for distributed systems is potentially superior when there is a considerable autonomy of participating entities: The common ontology for the application domain allows efficient building of interoperable systems with shared semantics of the communication. In addition, the agent coordination modelling approaches allow for specification and management of interactions among the participating 
entities (e.g., organizations or people). Moreover, the agent-based architectures provide for a common high-level schema on which to build future systems that are highly maintainable and manageable. Also, the autonomy of agents provides for a fundamentally new and potentially significant capability to have in the new electronic markets. The capability to automatically initiate actions may change the way that the manufacturing businesses collaborate and search for opportunities.

Second, the standards for information sharing and exchange in PCB/PCA industries (e.g., GenCAM) may benefit significantly from providing an agent-based service that would use the standard dictionary of the industry when providing data to suppliers. Different than the current established manner of sharing data among the customers and suppliers (which is based on complete file transfers), a GenCAM-based agent service would be able to provide information about the product on on-demand, asneeded basis. In this manner, a more manageable and agile way of information sharing is feasible.

Third, the agent-based approaches provide for modelling and synthesis of coordination policies for interacting autonomous entities. Such coordination policies provide basis for modelling interactions of collaborative, autonomous entities in business processes. An obvious example of the need that these agent-based approaches can fulfil is in the case of the RosettaNet standard. RosettaNet provides for Dictionaries, Framework, and Partner Interchange Process (PIP) definitions which, in turn, allow 'Dialog' among the partners (see Fig 3 taken from [1]). However, RosettaNet does not provide for capture of eBusiness processes. This is where the agent-based approaches can provide value to both an industry and standards making body as they provide high-level modelling and synthesis methodologies.

Last, the initial feedback from the workers in the manufacturing industry indicates that the agent-based approach could provide a desirable capability for flexible product information retrieval and supply chain information integration. Although preliminary, this feedback begins to validate our intuition in focusing on the two important areas for agent-based information systems: product information retrieval and supply chain information integration. 


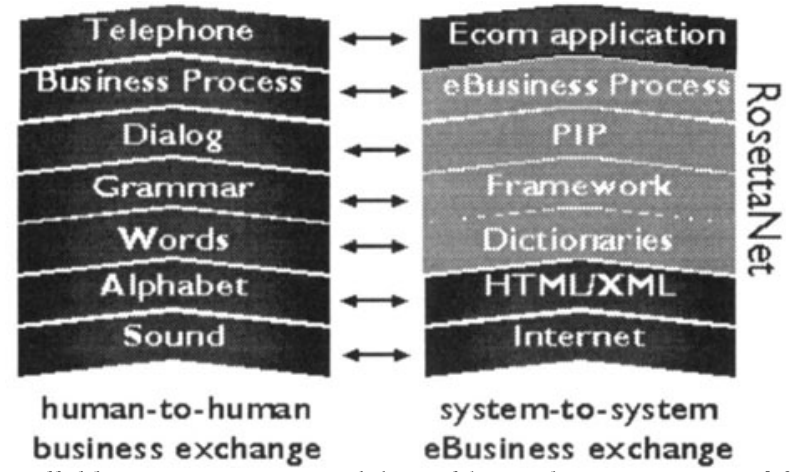

Figure 3 Parallel between RosettaNet deliverables and entities required for human-tohuman business exchange.

\section{CONCLUSION}

A need, an impact, and systems architecture analysis have been presented to address concerns within the Internet Commerce for Manufacturing (ICM) project. ICM is a National Institute of Standards and Technologies (NIST) sponsored project working with industry to create an environment where small manufacturers of mechanical and electronic components may participate competitively in virtual enterprises that manufacture printed circuit assemblies. The motivation and current work in adopting agent-oriented approaches for intra-enterprise information retrieval and inter-enterprise supply chain integration have been discussed in the context of emerging industrial standards.

\section{REFERENCES}

[1] GenCAM Standard Specification (2000). An Overview Document, http://www.gencam.org/overview/fact/fact.htm/

[2] RosettaNet Consortium (1999). An Overview Document, http://rosettanet.org/general/index_general.html/ 